\title{
Carbono de biomasa microbiana influenciada por los residuos de cinco especies de abonos verdes sobre un suelo bajo uso pecuario
}

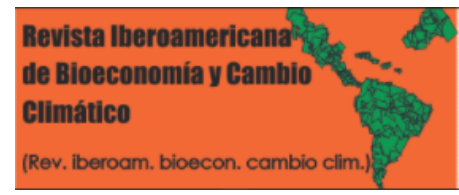

\section{Carbon of microbial biomass influenced by the residues of five species of green manures on a soil under livestock use}

\author{
Ruiz Alderete, David; Colque Arispe, Karina; Editor Académico Prof. \\ Dr. Angel Sol-Sánchez
}

\author{
David Ruiz Alderete \\ Centro de Investigación Agrícola Tropical CIAT, \\ Nicaragua \\ Karina Colque Arispe \\ kcolque@udabol.edu.bo \\ Centro de Investigación Agrícola Tropical CIAT, \\ Nicaragua \\ Editor Académico Prof. Dr. Angel Sol-Sánchez \\ Colegio de Postgraduados, México
}

Revista Iberoamericana de Bioeconomía y Cambio

\section{Climático}

Universidad Nacional Autónoma de Nicaragua, León, Nicaragua ISSN-e: 2410-7980

Periodicidad: Semestral

vol. 5, núm. 10, 2019

czuniga@ev.unanleon.edu.ni

Recepción: 15 Julio 2019

Aprobación: 04 Diciembre 2019

URL: http://portal.amelica.org/ameli/

jatsRepo/394/3941757004/index.html

DOI: https://doi.org/10.5377/ribcc.v5i10.8967

Autor de correspondencia: kcolque@udabol.edu.bo
Resumen: Se evaluó la influencia de residuos de abonos verdes (AV) sobre el Carbono (C) de la Biomasa microbiana del suelo (BMS) correspondiente a un área de uso pecuario (ganadero). El estudio fue desarrollado en el Proyecto Ganadería y Suelos del Centro de Investigación Agrícola Tropical (CIAT) Santa Cruz, Bolivia; el diseño experimental fue el de bloques completamente al azar, que contaba con 6 tratamientos y 4 repeticiones. La evaluación de la BMS fue a los 30 días de la incorporación de los residuos de las cinco especies de leguminosas empleadas como AV siendo Mucuna negra (Stizolobium aterrinum), Lab lab (Lablab purpureus), Mucuna ceniza (Stizolobium niveum), Crotalaria (Crotalaria juncea), Frejol de puerco (Canavalia ensiformis) y como testigo (vegetación espontánea) con el método de irradiación e incubación. Se encontró que las especies de AV tienen mayor concentración de C-BMS respecto al testigo, y entre los AV las especies de hábito rastrero son las que concentran mayor $\mathrm{C}$ en su BM.

Palabras clave: Abono verde, Carbono de biomasa microbiana, Leguminosas, Manejo sustentable del suelo.

Abstract: The influence of residues of green manure (AV) on Carbon (C) of soil microbial biomass (BMS) corresponding to an area of livestock use (livestock) was evaluated. The study was developed in the Livestock and Soil Project of the Tropical Agricultural Research Center (CIAT) Santa Cruz, Bolivia; The experimental design was completely randomized, with 6 treatments and 4 repetitions. The evaluation of the BMS was 30 days after the incorporation of the residues of the five legume species used as AV being black Mucuna (Stizolobium aterrinum), Lab lab (Lablab purpureus), Mucuna ash (Stizolobium niveum), Crotalaria (Crotalaria juncea), pork bean (Canavalia ensiformis) and as a control (spontaneous vegetation) with the irradiation and incubation method. It was found that the AV species have the highest concentration of C-BMS with respect to the control, and among the AV the species of creeping habit are those that concentrate the highest $\mathrm{C}$ in their BM.

Keywords: Green manure, Carbon of microbial biomass, Legumes, Sustainable land management. 


\section{INTRODUCCIÓN}

Según Vallejo (2013) menciona "en la actualidad las propiedades biológicas se han convertido en criterios importantes para valorar el manejo o uso de los suelos, de tal forma que se crea la necesidad de orientar la producción agropecuaria hacia nuevas tecnologías fundamentadas en la recuperación de los suelos degradados a través de un manejo agroecológico sostenido que favorezca la biodiversidad".

Es bien conocido que la ganadería o pecuaria en Bolivia se ha desarrollado de manera extensiva, destinándose grandes terrenos, con bajos niveles de inversión y manejo inadecuado de praderas, además algunas de las áreas destinadas a la actividad son el resultado de un cambio de uso de suelo los cuales son derivados de áreas agrícolas, en la que de igual forma se presento la ausencia de un manejo sustentable del recurso. Para Mahecha et al. (2002) la falta del manejo apropiado al suelo provocada un deterioro ambiental de los ecosistemas y un impacto negativo en los sectores socioeconómicos de un país.

De acuerdo con Armida et al. (2005) la biomasa microbiana medida como C biomásico se puede utilizar como un indicador sensible al manejo ya que detectan tempranamente el impacto de las prácticas de manejo agrícola. Por esta razón es necesario emplear prácticas preventivas como fomentar el uso de los residuos de cosecha, empleo de plantas de cobertura lo cual poco a poco va tomando importancia entre los productores.

\section{MATERIALES Y MÉTODO}

Localización: Se estableció el ensayo en la Estación Experimental Agrícola de Saavedra (EEAS) ubicada en el Municipio General Saavedra, en la provincia Obispo Santiesteban, distante aproximadamente a $65 \mathrm{Km}$. de

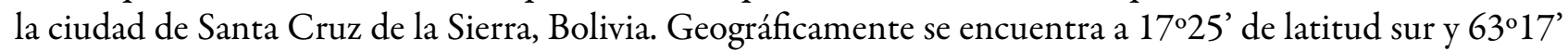
de longitud oeste, y a 295 m.s.n.m. Se caracteriza por presentar una precipitación media anual de $1352 \mathrm{~mm}$ y una temperatura media de $24.2^{\circ} \mathrm{C}$.

Factor bajo estudio: Se evaluó la influencia de la descomposición de residuos de cinco especies de abonos verdes (AV) sobre el carbono de la biomasa microbiana (C-BM) en un suelo de uso pecuario.

Diseño experimental: Se evaluaron 6 tratamientos utilizando un diseño de bloques completamente al azar, con cuatro repeticiones para un total de 24 unidades experimentales.

Descripción de los tratamientos, tabla 1: Los tratamientos de AV fueron mucuna negra (Stizolobium aterrinum), Lab (Lablab purpureus), mucuna ceniza (Stizolobium niveum), Crotalaria (Crotalaria juncea), Frejol de puerco (Canavalia ensiformis) y como testigo vegetación espontanea (maleza).

NotAS DE AUTOR

kcolque@udabol.edu.bo 
TABLA 1

Descripción de los tratamientos

\begin{tabular}{ll}
\hline Tratamiento & Descripción \\
\hline 1 & Frejol de puerco \\
2 & Lab lab \\
3 & Mucuna ceniza \\
4 & Mucuna negra \\
5 & Crotalaria \\
6 & Testigo \\
\hline
\end{tabular}

Elaboracion propia

Manejo del experimento: Luego de 3 meses de desarrollo vegetativo de las especies de AV se procedió a realizar el manejo de su biomasa foliar, realizando la corta y la incorporación en labranza mínima a $5 \mathrm{~cm}$ de profundidad, posteriormente a los 30 días de realizado el manejo de la cobertura se tomaron muestras de suelo de los tratamientos para determinar las concentraciones de C-BMS.

Las muestras fueron extraídas con un barreno de $2 \mathrm{~cm}$ de diámetro a una profundidad de $0-20 \mathrm{~cm}$, se tomaron 10 submuestras por parcela, se conformó una muestra compuesta de cada unidad experimental. Estas fueron acondicionadas en bolsas plásticas y transportadas en cajas de plastoform con hielo para disminuir la actividad microbiana. En laboratorio fueron mantenidas en cámara fría $\left(8^{\circ} \mathrm{C}\right)$ por 1 día.

Las muestras se secaron al aire por $24 \mathrm{hrs}$, se homogeneizaron con un tamiz de $4 \mathrm{~mm}$, retirando los restos vegetales. De cada muestra se determinó de la humedad gravimétrica. Los resultados fueron calculados tomando como base la masa de suelo seco.

En la humedad gravimétrica; el contenido gravimétrico de agua de las muestras de suelo fue determinada por la pérdida de peso al secarlas a $105^{\circ} \mathrm{C}$ en estufa por $24 \mathrm{hrs.} \mathrm{(Cochran} \mathrm{y} \mathrm{Barber} \mathrm{1993).} \mathrm{Estas} \mathrm{mediciones}$ se realizaron para obtener el factor de corrección de la humedad en el cálculo de los valores de biomasa microbiana. Evaluación de la biomasa microbiana (carbono microbiano), la metodología empleada para la evaluación del flujo de $\mathrm{CO}_{2}$ debido a la respiración de los microorganismos del suelo está basada en las conclusiones de Parkinson y Paul (1982), quienes indican que el Carbono (C) de los microorganismos muertos es más rápidamente mineralizado. De manera que al provocar su muerte por irradiación o fumigación en la muestra de suelo que los contiene, y luego reinocularla con una porción de la misma muestra, se obtiene una estimación aproximada del tamaño de la biomasa microbiana del suelo.

La evaluación del C de la biomasa microbiana fue realizada por irradiación - incubación, se utilizó el procedimiento descrito por Ferreira et al. (1999), y posterior incubación, siguiendo el método desarrollado por Parkinson y Paul (1982).

De cada muestra se retiraron 4 submuestras de 200 gr de suelo (base húmeda) dos, fueron irradiadas en horno microondas por 2 min (energía de trabajo $(J)=1.62 \times 10^{5}, J=1350 \mathrm{~W} \times 120 \mathrm{~s}$ ), estas fueron reinoculadas con $1 \mathrm{~g}$ de la muestra original e incubadas en frascos de $1 \mathrm{~L}$. de capacidad, herméticamente cerrados, dentro 
de los frascos fueron colocados frascos de $200 \mathrm{ml}$ de capacidad con $25 \mathrm{ml} \mathrm{de} \mathrm{NaOH}$ (1N), para la absorción de $\mathrm{CO}_{2}$ proveniente de la actividad microbiana. Las restantes tres muestras, fueron colocadas a incubar de la misma manera que las muestras irradiadas. Por otro lado, se colocó $\mathrm{NaOH}(1 \mathrm{~N})$ en frascos sin suelo. Transcurridos 10 días, se tomaron los frascos con $\mathrm{NaOH}$, procediéndose a la titulación con $\mathrm{HCl}(1 \mathrm{~N})$, previo agregado al $\mathrm{NaOH}$, de $5 \mathrm{ml} \mathrm{de} \mathrm{BaCl}_{2}$ al $2 \%$ y tres gotas de fenolftaleína. Se registró el gasto de $\mathrm{HCl}$ hasta la desaparición del color rosa, según detalla Frioni, (1990).

Los resultados de la biomasa microbiana se expresaron como ec1 , 2 :

$$
\operatorname{Biomasa}\left(\mu g C-\mathrm{CO}_{2} g^{-1} \text { sueloseco }\right)=\frac{x-y}{k}
$$

Donde: $\mathrm{X}=\mu \mathrm{g}$ de $\mathrm{C}-\mathrm{CO}_{2} 100-1 \mathrm{~g}$

$\mathrm{Y}=\mu \mathrm{g}$ de $\mathrm{C}-\mathrm{CO}_{2} 100-1 \mathrm{~g}$ suelo seco (1), incubado

$\mathrm{k}=$ Constante 0.45 , que representa la fracción de la biomasa total del suelo que es mineralizada en condiciones controladas.

$$
\text { (1) } m g \mathrm{de} C-\mathrm{CO}_{2} \mathrm{~kg}^{-1} \text { sueloseco }=\frac{(\mathrm{GB}-\mathrm{GM}) \mathrm{N}(\mathrm{HCL}) 0.0006}{\text { peso de la muestra seca }(\mathrm{g}) \times 1000000}
$$

$\mathrm{GB}=$ gasto de $\mathrm{HCl}$ del blanco (sin suelo)

$\mathrm{GM}=$ gasto de $\mathrm{HCl}$ de la muestra

$\mathrm{N}(\mathrm{HCL})=$ normalidad del $\mathrm{HCl}$

0.006 = número atómico de $\mathrm{C} / 1000$ (para obtener $m g$ )

Análisis de datos: A los datos obtenidos de la variable estudiada se le realizo el análisis de varianza y como prueba de significancia se empleo la Diferencia Mínima Significativa (DMS) al 5\% de probabilidad. El programa utilizado para el análisis de varianza y comparación de medias fue el Info Stat versión 2015(Ver tabla 2Anexos).

\section{RESULTADOS Y DISCUSióN}

\section{Biomasa microbiana (Cmic)}

La Biomasa Microbiana del Suelo (BMS) evaluada por el contenido de $\mathrm{C}_{\text {mic }}$ vario significativamente $(\mathrm{P}<0.05)$ con respecto a los tratamientos con residuos de AV. El promedio general es de $5.90 \mathrm{mg} \mathrm{C}_{\text {mic }} \mathrm{kg}^{-1}$ y el Coeficiente de Variación de 7.98 \%. Siendo el tratamiento de Lab lab que promovió mayor contenido de $\mathrm{C}$ respecto a los demás tratamientos, haciendo una comparación con el testigo $\left(2.84 \mathrm{mg} \mathrm{C}_{\mathrm{mic}} \mathrm{kg}^{-1}\right)$ el cual obtuvo menor BMS este tratamiento fue superior en $194 \%$. Aun así Lab lab (8.34 mg $\mathrm{C}_{\text {mic }} \mathrm{kg}^{-1}$ ) no difiere estadísticamente con Mucuna negra $\left(7.74 \mathrm{mg} \mathrm{C}_{\text {mic }} \mathrm{kg}^{-1}\right)$. Seguidamente se tiene a Mucuna ceniza, Crotalaria y Frejol de puerco con 6.37, 5.90, $4.26 \mathrm{mg} \mathrm{C}_{\text {mic }} \mathrm{kg}^{-1}$ respectivamente (Figura 1). 


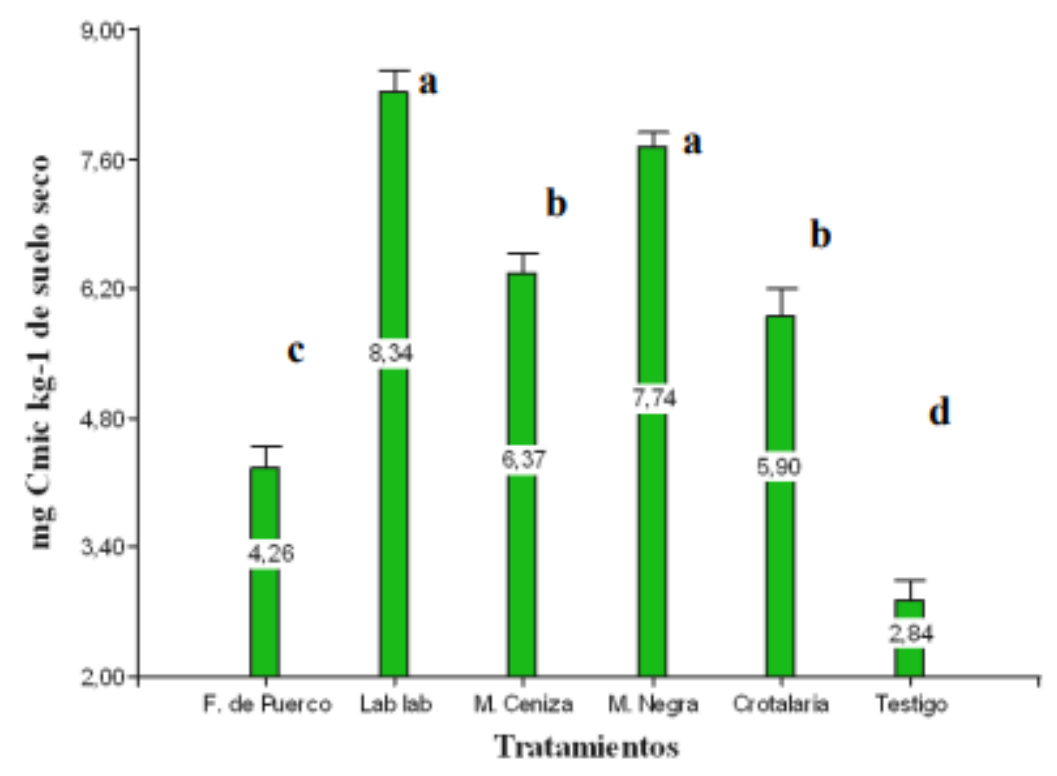

FIGURA 1

Contenido de $\mathrm{C}$ en la Biomasa Microbiana por la incorporación de cinco especies de AV

La concentración de la BMS anteriormente descrita se debe a la manutención de residuos vegetales sobre el suelo que independiente de la composición de los residuos promueve aumento de la actividad de los microorganismos heterotróficos del suelo (CASTILHOS et al., 2004). Para Negri et al. (2013) la actividad de la biomasa microbiana del suelo hace posible la descomposición de los diferentes materiales orgánicos, condiciona las transformaciones de nitrógeno y carbono en el suelo y torna disponibles los nutrientes para las plantas, por otra parte Gama-Rodrigues y Gama-Rodrigues (1999) indican ellos utilizan esos materiales como fuente de nutrientes y energía para la formación y desenvolvimiento de sus células.

Por otra parte Beltran et al. (2006) menciona con el objetivo de la recuperación de fertilidad en un suelo árido con pobres características nutrimentales y contenido de MO del 0,54\%, empleo Lab lab al ser este una especie leguminosa de amplia aceptación en Centroamérica como abono verde en agroecosistemas conservacionista, los autores evaluaron la producción de $\mathrm{CO}_{2}$ de la biomasa microbiana respecto al manejo de la biomasa foliar con incorporación y sin incorporación de la especie, también el comportamiento de este en dos fechas de muestreo, encontrando que hay mayor cantidad de $\mathrm{C}$ mineralizado a los 60 días después de la incorporación $\left(22,54 \mathrm{C} \mathrm{kg \cdot día}{ }^{-1}\right.$, siendo que en el primer muestro a los 30 días la concentración fue de 16,50 C kg.día ${ }^{-1}$. En relación con el manejo realizado la mayor cantidad de $\mathrm{C}$ se encontró en los residuos incorporados $23,92 \mathrm{C} \mathrm{kg} \cdot \mathrm{di}^{-1}$.

De acuerdo a Sanclemente (2009) encontró similares resultados mencionando que la mayor reducción

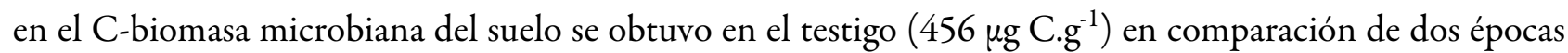
de muestreo, el establecimiento del abono verde (Mucuna pruriens) minimizó esa reducción en 26\% (119 $\left.\mu \mathrm{g} \mathrm{C.g}{ }^{-1}\right)$, mostrando un efecto positivo sobre el incremento en las poblaciones microbianas del suelo, con respecto al testigo.

Considerando que la BMS es la fracción más activa de $\mathrm{MO}$, este compartimiento es el responsable por la inmovilización temporaria de $\mathrm{C}, \mathrm{N}, \mathrm{P}, \mathrm{K}, \mathrm{Ca}, \mathrm{Mg}, \mathrm{S}$ y micronutrientes, los cuales son liberados después de su muerte y descomposición, tornándose disponibles para las plantas mencionan Gama-Rodrigues y GamaRodrigues (1999), de modo que se tendrá menores pérdidas en el sistema suelo-planta (Mercante, 2001).

Negri et al. (2013) observaron entre los abonos verdes y las fechas de muestreo un coeficiente de correlación de $83 \%$ en una regresión lineal, lo que indica la disminución gradual de la respiración microbiana en el tiempo. 
En el mismo experimento los autores encontraron que en el sistema convencional la actividad microbiana es variable, siendo mayor en la leguminosa a los 30 días, en la gramínea a los 60 días y en la mezcla de gramínea y leguminosa a los 90 días de realizado el manejo de los abonos verdes, por el contrario la actividad microbiana se ve aumentada en la mezcla de gramínea y leguminosa en el sistema de siembra directa.

En un estudio desarrollado por Mercante et al. (2008) sobre un Argissolo Vermelho encontraron resultados contrarios a los del presente estudio en donde el tratamiento de maleza ( $139.7 \mu \mathrm{g} \mathrm{C} \mathrm{g}{ }^{-1}$ suelo seco) fue superior en el contenido de C-BMS respecto al tratamiento de mucuna ceniza ( $103.0 \mu \mathrm{g} \mathrm{C} \mathrm{g} \mathrm{g}^{-1}$ suelo seco) en siembra directa, y por lo que se refiere a la labranza convencional ( $93.2 \mu \mathrm{g} \mathrm{C} \mathrm{g}$ suelo seco) este fue inferior al tratamiento de mucuna.

Esta tendencia de aumento de la actividad microbiana generada a partir de la adición de los residuos, coinciden con lo reportado por Mercante (2001) que menciona que tanto la cantidad como la calidad de los residuos vegetales en los sistemas productivos provocan alteraciones en la composición de la comunidad microbiana influenciando su tasa de descomposición.

Si bien la sustitución de vegetación nativa por sistemas agrícolas cultivados repercute en las pérdidas de nutrientes; de esa forma se desenvuelve el proceso de degradación química, física y biológica del suelo, teniendo como producto la reducción de productividad de los cultivos cada vez más acentuada con el manejo inadecuado y el uso continuo del suelo, tales detrimentos son debidos al tipo de sistema adoptado (Da Silva et al., 2006), por tanto vale decir que el manejo sostenible del suelo a través del empleo de especies de cobertura como abono verde resulta favorable para contrarrestar la situación ya sea conservando o incrementando la concentración de nutrientes.

\section{Conclusión}

Las especies con hábito de crecimiento rastrero fueron en las que se observaron mayor contenido de BMS en el siguiente orden decreciente la especie Lab, Mucuna negra, Mucuna ceniza, y por consiguiente se establece que la presencia de residuos vegetales influencia la microbiota del suelo, demostrando que el uso de plantas de cobertura en suelos de uso pecuario favorece la actividad microbiana y reduce la pérdida de nutrientes debido a la inmovilización por otro lado vale decir que también interviene en el ciclaje de nutrientes.

Finalmente los residuos de abonos verdes dentro del manejo sostenible del suelo son fuente de energía y nutrientes para los microorganismos del suelo, siendo una alternativa para contrarrestar el continuo deterioro que sufren las áreas de uso pecuario ganadero.

\section{LITERATURA CITADA}

Armida, L; Espinosa, V; Palma, D; Galvis, A; Salgado, S. (2005). Carbono en biomasa microbiana y carbono soluble como indicadores de calidad de vertisoles cultivados con caña azucarera. Terra Latinoamericana, 23(4), 545-551.

Cochrane, T. T., \& Barber, R. G. (1993). Análisis de suelos y plantas tropicales (No. 631.41 C659a). Centro de Investigación Agrícola Tropical.

Beltrán, F; García, J. L; Valdez, R; Murillo, B; Troyo, E; Larrinaga, J. A; Beltrán, L. F. (2006). Efecto del sistemas de labranza e incorporación de abono verde (Lablab purpureus) sobre la respiración edáfica en un Yermosol háplico.. Recuperado 18 de agosto de 2016, a partir de http://www.redalyc.org/pdf/339/33911412.pdf

Ferreira, A. D. S., Camargo, F. A. D. O., \& Vidor, C. (1999). Utilização de microondas na avaliação da biomassa microbiana do solo. Revista Brasileira de Ciência do solo, 23(4), 991-996.

Frioni, L. (1990). Ecología microbiana del suelo. Departamento de Publicaciones y Ediciones de la Universidad de la República, Montevideo, Uruguay. 519p 
Gama-Rodrigues, A. C., y Gama Rodrigues, E. F. (1999). Biomassa microbiana e ciclagem de nutrientes. Fundamentos da matéria orgânica do solo: ecossistemas tropicais e subtropicais. Gênesis Edições. Porto Alegre, Brasil, p 227-243.

Mahecha, L; Gallego, L. A; Peláez, F. J. (2002). Situación actual de la ganadería de carne en Colombia y alternativas para impulsar su competitividad y sostenibilidad. Revista Colombiana de Ciencias Pecuarias, 15(2), 213-225.

Mercante, F. M. (2001). Os microrganismos do solo e a dinâmica da matéria orgânica em sistemas de produção de grãos e pastagem. Embrapa Agropecuária Oeste-Sistema de Produção (INFOTECA-E).

MERCANTE, F. M., SILVA, R. F. D., FRANCELINO, C. S. F., CAVALHEIRO, J. C. T., \& OTSUBO, A. A. (2008). BIOMASSA MICROBIANA, EM UM ARGISSOLO VERMELHO, EM DIFERENTES COBERTURAS VEGETAIS, EM ÁREA CULTIVADA COM MANDIOCA. ACTA SCIENTIARUM. AGRONOMY, 30(4), 479-485.

Negri, L. A. B., Samaniego, M. D. P. G., \& Roa, M. A. K. M. (2013). EVALUACIÓN DE LA ACTIVIDAD MICROBIANA EN FUNCIÓN DE CUATRO ABONOS VERDES EN UN RHODIC KANDIUDOX BAJO SIEMBRA DIRECTA Y LABOREO CONVENCIONAL. Investigación Agraria, 7(1), 32-41.

Parkinson, D; Paul, E.A. (1982). Microbial biomasa. Methods of soil analysis. Part 2. Chemical and Microbiological Properties. Second Edition. Madison, Wisconsin, USA. p821 - 830.

Sanclemente, O. (2009). Efecto del cultivo de cobertura: Mucuna pruriens, en algunas propiedades físicas, químicas y biológicas de un suelo Typic Haplustalfs, cultivado con maíz (Zea Mays L.) en zona de ladera del municipio de Palmira, Valle. Universidad Nacional de Colombia Sede Palmira. Tesis de Maestría. Recuperado a partir de http://www.bdigital.unal.edu.co/712/

CASTILHOS, D. D., Santos, V., Castilhos, R., Pauletto, E., Gomes, A., \& Silva, D. (2004). Biomassa, atividade microbiana e teores de carbono e nitrogênio totais de um planossolo sob diferentes sistemas de manejo. Current Agricultural Science and Technology, 10(3).

Da Silva, X. F. A; Maia, S. M. F; de Oliveira, T. S; \& de Sá Mendonça, E. (2006). Biomassa microbiana e matéria orgânica leve em solos sob sistemas agrícolas orgânico e convencional na Chapada da Ibiapaba-CE. Revista Brasileira de Ciência do Solo, 30(2), 247-258.

Vallejo, V. (2013). Importancia y utilidad de la evaluación de la calidad de suelos mediante el componente microbiano: experiencias en sistemas silvopastoriles. Colombia Forestal, 16(1), 83-99. 
Anexo

TABLA 2

Análisis de varianza efecto de abonos verdes sobre el C-BMS

\begin{tabular}{|c|c|c|c|c|}
\hline ble & $\mathrm{N}$ & $\mathrm{R}^{2}$ & $\mathrm{R}^{2} \mathrm{Aj}$ & $\mathrm{CV}$ \\
\hline rbono de BMS & & 24 & 0,97 & 0,95 \\
\hline
\end{tabular}

Cuadro de Análisis de la Varianza (SC tipo III)

\begin{tabular}{lccccc}
\multicolumn{1}{c}{ F.V. } & SC & gl & CM & F & p-valor \\
\hline Modelo. & 87,24 & 8 & 10,90 & 54,39 & $<0,0001$ \\
Bloques & 0,76 & 3 & 0,25 & 1,27 & 0,3200 \\
Tratamientos & 86,47 & 5 & 17,29 & 86,26 & $<0,0001$ \\
Error & 3,01 & 15 & 0,20 & & \\
Total & 90,25 & 23 & & & \\
\hline
\end{tabular}

Test:LSD Fisher Alfa $=0,05$ DMS $=0,67485$

Error: 0,2005 gl: 15

Tratamientos Medias $\mathrm{n} \quad$ E.E.

\begin{tabular}{lllll}
\hline Lab lab & 8,34 & 4 & 0,22 & A
\end{tabular}

M. Negra $\quad 7,74 \quad 4 \quad 0,22 \quad$ A

$\begin{array}{lllll}\text { M. Ceniza } & 6,37 & 4 & 0,22 & \text { B }\end{array}$

$\begin{array}{lllll}\text { Crotalaria } & 5,90 & 4 & 0,22 & \text { B }\end{array}$

$\begin{array}{lllll}\text { F. de Puerco } & 4,26 & 4 & 0,22 & \text { C }\end{array}$

\begin{tabular}{lllll} 
Testigo & 2,84 & 4 & 0,22 & D \\
\hline
\end{tabular}

Medias con una letra común no son significativamente diferentes $(p>0,05)$ 